\title{
ON THE EVALUATION OF INDEFINITE INTEGRALS INVOLVING THE SPECIAL FUNCTIONS: DEVELOPMENT OF METHOD*
}

\author{
BY \\ L. C. MAXIMON AND G. W. MORGAN \\ Brown University
}

Summary. The solution of a general second order linear partial differential equation in two variables by Laplace transforms is utilized to develop a method for the evaluation of a large class of indefinite integrals involving the special functions.

1. Introduction. Indefinite integrals involving the special functions are of importance in analysis and in many physical problems. Although extensive work has been done on integrals containing the Bessel functions the literature contains relatively few results for any of the other special functions. For references see the paper immediately :succeeding this one in this issue. ${ }^{1}$ In the following we develop a method of evaluating integrals which is equally applicable to many of the special functions, including the Bessel functions as a particular case.

2. Motivation. Let us consider the solution of a linear homogeneous partial differential equation in one space variable and time which is typical of those encountered in mathematical physics:

$$
\frac{\partial y}{\partial t}=\frac{\partial}{\partial x}\left[a(x) \frac{\partial y}{\partial x}\right] .
$$

We may solve this equation by use of the Laplace transform defined by

$$
Y(x, s)=\int_{0}^{\infty} e^{-s t} y(x, t) d t
$$

in terms of whieh Eq. (1) may be written

$$
a(x) Y^{\prime \prime}+a^{\prime}(x) Y^{\prime}-s Y=-y(x, 0),
$$

where primes denote derivatives with respect to $x$. The ordinary inhomogeneous differential equation (3) may be solved by variation of parameters, giving

$$
Y(x, s)=Y_{1} \int \frac{Y_{2} y(x, 0)}{a(x) W(x)} d x-Y_{2} \int \frac{Y_{1} y(x, 0)}{a(x) W(x)} d x
$$

in which $Y_{1}$ and $Y_{2}$ are any two linearly independent solutions of the homogeneous equation

$$
a(x) Y^{\prime \prime}+a^{\prime}(x) Y^{\prime}-s Y=0
$$

and

$$
W(x)=Y_{1} Y_{2}^{\prime}-Y_{1}^{\prime} Y_{2}
$$

is the Wronskian of $Y_{1}$ and $Y_{2}$. Thus, assuming that the boundary and initial conditions pertaining to Eq. (3) are known, the limits on the integrals in Eq. (4) may be determined

*Received April 20, 1954.

${ }^{1}$ L. C. Maximon, $O n$ the evaluation of indefinite integrals involving the special functions: application of method, quoted as $[\mathrm{B}]$ in the following. 
and the transform $Y(x, s)$ of the solution is then given in terms of indefinite integrals of known functions.

Rather than proceeding to the inversion of $Y(x, s)$, however, we shall consider the integrals in Eq. (4) as our unknowns and hence would like to have $Y(x, s)$ a known solution. One case in which the solution is quite simple is provided by assuming the boundary and initial conditions to be such that the solution $y(x, t)$ is actually time. independent, so that

and $y_{0}$ satisfies

$$
\begin{aligned}
& y(x, t)=y(x, 0) \equiv y_{0}, \\
& Y(x, s)=\frac{y(x, 0)}{s}
\end{aligned}
$$

$$
\left[a(x) y^{\prime}\right]^{\prime}=0 .
$$

The particular boundary conditions that must be chosen need not concern us here, since, as will be seen later, they do not enter the final formulas. Writing

$$
I_{i}=s \int \frac{Y_{i} y_{0}}{a(x) W(x)} d x \quad i=1,2,
$$

we now solve for $I_{i}$ from Eqs. (4) and (7):

$$
Y_{1} I_{2}-Y_{2} I_{1}=y_{0} \text {. }
$$

Differentiation gives

Hence

$$
Y_{1}^{\prime} I_{2}-Y_{2}^{\prime} I_{1}=y_{0}^{\prime}
$$

$$
I_{i}=\frac{y_{0} Y_{i}^{\prime}-y_{0}^{\prime} Y_{i}}{W} \quad i=1,2
$$

and from Eqs. (9) and (11) we have

$$
s \int Y_{i} y_{0} d x=a(x)\left[y_{0} Y_{i}^{\prime}-y_{0}^{\prime} Y_{i}\right] \quad i=1,2
$$

afier substituting

$$
W=\text { const. } \times \exp \left[-\int \frac{a^{\prime}(x)}{a(x)} d x\right]=\frac{\text { const. }}{a(x)}
$$

from Eq. (3). By differentiating Eq. (12) it may be noted that this equation is valid. for any solutions $Y_{i}$ and $y_{0}$ of Eqs. (5) and (8) respectively.

Let us consider a particular example. With

we may set

$$
a(x)=1-x^{2}, \quad s=-\nu(\nu+1)
$$

$$
Y_{i}=P_{v}(x),
$$

the Legendre function, and from Eq. (8)

$$
y_{0}=A+B \log \left|\frac{1+x}{1-x}\right|,
$$


where $A$ and $B$ are arbitrary constants. Substituting Eqs. (14), (15) and (16) in Eq. (12) we obtain two indefinite integrals involving the Legendre function. For $A=1$, $B=0$, we have

$$
-\nu(\nu+1) \int P_{\nu}(x) d x=\left(1-x^{2}\right) P_{\nu}^{\prime}(x),
$$

and for $A=0, B=1$

$-\nu(\nu+1) \int P_{\nu}(x) \log \left|\frac{1+x}{1-x}\right| d x=\left(1-x^{2}\right)\left[P_{\nu}^{\prime}(x) \log \left|\frac{1+x}{1-x}\right|-\frac{\frac{1}{2} P_{\nu}(x)}{1-x^{2}}\right]$.

3. Derivation of generalized formula. We now extend Eq. (12) by considering a more general equation than Eq. (1), and hence let $y(x, t)$ be a solution of

$$
\sum_{i=0}^{2}\left[a_{i}(x)+b_{i}(x) \frac{\partial}{\partial t}\right] \frac{\partial^{i} y}{\partial x^{i}}=0,
$$

in which $a_{i}(x)$ and $b_{i}(x)$ are arbitrary. Introducing higher derivatives with respect to $t$ does not seem to generalize further the final results. Additional results may be obtained, however, by considering an equation containing higher derivatives with respect to $x$ and this is done in [B]. Defining $Y(x, s)$ as in Eq. (2) and taking the Laplace transform of Eq. (19) with respect to $t$ we obtain

$$
\sum_{i=0}^{2}\left[a_{i}(x)+s b_{i}(x)\right] \frac{d^{i} Y}{d x^{i}}=\sum_{i=0}^{2} b_{i}(x) \frac{d^{i} y_{0}}{d x^{i}} \equiv K(x),
$$

which we solve by variation of parameters, giving

$$
Y(x, s)=Y_{1} \int \frac{Y_{2} K(x)}{\left(a_{2}+s b_{2}\right) W}-Y_{2} \int \frac{Y_{1} K(x)}{\left(a_{2}+s b_{2}\right) W} d x .
$$

Here $Y_{1}$ and $Y_{2}$ are any two linearly independent solutions of the homogeneous equation

$$
\sum_{i=0}^{2}\left[a_{i}(x)+s b_{i}(x)\right] \frac{d^{i} Y}{d x^{i}}=0
$$

and $W$ is the Wronskian of $Y_{1}$ and $Y_{2}$, defined in Eq. (6). We again assume that the boundary and initial conditions are such that $y(x, t)$ is independent of time, so that Eq. (7) is valid and $y_{0}$ is a solution of

$$
\sum_{i=0}^{2} a_{i}(x) \frac{d^{i} y}{d x^{i}}=0
$$

Substituting Eq. (7) in Eq. (21) we have

$$
y_{0}=Y_{1} I_{2}-Y_{2} I_{1},
$$

where

$$
\begin{gathered}
I_{i}=s \int \frac{Y_{i} K(x)}{c_{2} W} d x \quad i=1,2, \\
c_{i}=a_{i}(x)+s b_{i}(x) \quad i=0,1,2 .
\end{gathered}
$$


As in Eqs. (10), (11) and (12) we differentiate Eq. (24), solve for $I_{i}$, and equate the result to the right hand side of Eq. (25a). In this equation we may then substitute

$$
W=\text { const. } \times \exp \left[-\int\left(\frac{c_{1}}{c_{2}}\right) d x\right]
$$

and

$$
s K(x)=\sum_{i=0}^{2}\left(c_{i}-a_{i}\right) \frac{d^{i} y_{0}}{d x^{i}}
$$

from Eqs. (20) and (25b), obtaining finally

$$
\int Y_{1} \exp \left[\int\left(\frac{c_{1}}{c_{2}}\right) d x\right] \cdot \frac{1}{c_{2}} \sum_{i=0}^{2}\left(c_{i}-a_{i}\right) \frac{d^{i} y_{0}}{d x^{i}} d x=\left(y_{0}^{\prime} Y_{1}-y_{0} Y_{1}^{\prime}\right) \exp \left[\int\left(\frac{c_{1}}{c_{2}}\right) d x\right]
$$

and a similar and redundant equation with $Y_{2}$ in place of $Y_{1}$. In Eq. (28) $Y_{1}$ is a solution of

$$
\sum_{i=0}^{2} c_{i} \frac{d^{i} Y}{d x_{i}}=0 .
$$

Also, $y_{0}$ is a solution of Eq. (23), in view of which Eq. (28) may also be written in the form

$$
\int Y_{1} \exp \left[\int\left(\frac{c_{1}}{c_{2}}\right) d x\right] \cdot \frac{1}{c_{2}} \sum_{i=0}^{2} c_{i} \frac{d^{i} y_{0}}{d x^{i}} d x=\left(y_{0}^{\prime} Y_{1}-y_{0} Y_{1}^{\prime}\right) \exp \left[\int\left(\frac{c_{1}}{c_{2}}\right) d x\right] \text {. }
$$

Moreover, in Eq. (30) $y_{0}$ may be completely arbitrary; Eq. (23) need no longer be assumed. Frequently, however, it will be found more convenient to use Eq. (28), in which case Eq. (23) must be satisfied. By differentiating it may be noted that Eq. (28) is valid for any solutions $Y_{1}$ and $y_{0}$ of Eqs. (29) and (23) respectively, and Eq. (30) is valid for any solution $Y_{1}$ of Eq. (29) and arbitrary $y_{0}$. Equations (28) and (30) are the desired generalizations of Eq. (12). Their use will be illustrated later by some examples.

We are indebted to Dr. Paul Germain for pointing out to us that Eq. (30) may also be obtained by considering the Wronskian

$$
W^{*} \equiv Y_{1} y_{0}^{\prime}-Y_{1}^{\prime} y_{0},
$$

where $Y_{1}$ is a solution of Eq. (29) and $Y_{0}$ is arbitrary. Thus, if we let

$$
f(x)=c_{2} y_{0}^{\prime \prime}+c_{1} y_{0}^{\prime}+c_{0} y_{0},
$$

then

$$
c_{2} W^{* \prime}+c_{1} W^{*}=f Y_{1},
$$

and solving for $W^{*}$ we have

$$
W^{*} \exp \left[\int\left(\frac{c_{1}}{c_{2}}\right) d x\right]=\int \frac{f(x) Y_{1}}{c_{2}} \exp \left[\int\left(\frac{c_{1}}{c_{2}}\right) d x\right] d x,
$$

which is identical with Eq. (30). Although this derivation is far simpler than that given in arriving at Eqs. (28) and (30), the lengthier discussion is presented for a number of 
reasons. First, it is in Eq. (28) that one sees clearly that to obtain fairly simple integrands (and hence useful integrals) one must choose $Y_{1}$ and $y_{0}$ so that all but one of the factors $c_{i}-a_{i}$ is zero, and this equation is only put in evidence by the first method of derivation. Moreover, although relations involving the Wronskian have often been used in the past to evaluate indefinite integrals, the exhibition of Eq. (30) solely as an expression for the Wronskian $W^{*}$ does not point up the possibility of immediately adapting this equation to the evaluation of a very general class of useful integrals. Second, the idea of starting with a relatively difficult time dependent problem leading to a solution in terms of integrals which must be calculated, and then looking for a particularly simple solution (e.g. a time independent solution) which may be arrived at by other means, thus enabling us to reverse the usual role of known and unknown quantities in the time dependent solution, may prove fruitful in connection with other problems.

While extensive work has been done on indefinite integrals involving the Bessel functions, many of which will be obtained by the methods developed here in [B], we give two examples of other integrals to illustrate the simplicity of the present procedure. ${ }^{2}$

Thus if we choose $Y_{1}=W_{k, \mu}$, the Whittaker function which is a solution of

$$
Y^{\prime \prime}+\left(-\frac{1}{4}+\frac{\kappa}{x}+\frac{\frac{1}{4}-\mu^{2}}{x^{2}}\right) Y=0,
$$

and if we let $y_{0}=W_{x, \nu}$, then from Eq. (28) we have immediately

$$
\left(\nu^{2}-\mu^{2}\right) \int x^{-2} W_{\kappa, \mu} W_{\kappa, \nu} d x=W_{\kappa, \nu}^{\prime} W_{\kappa, \mu}-W_{\kappa, \nu} W_{\kappa, \mu}^{\prime} .
$$

On the other hand, if we let $y_{0}=W_{\lambda, \mu}$, then again from Eq. (28) we have

$$
(\kappa-\lambda) \int x^{-1} W_{\alpha, \mu} W_{\lambda, \mu} d x=W_{\lambda, \mu}^{\prime} W_{\alpha, \mu}-W_{\lambda, \mu} W_{\kappa, \mu}^{\prime} .
$$

Equations (31) and (32) were arrived at by choosing $Y_{1}$ and $y_{0}$ so that $\sum_{i}\left(c_{i}-a_{i}\right) d^{i} y_{0} / d x^{i}$ would reduce to a single term. Evidently many integrals may be evaluated in this way, but the class of integrals which may be obtained by this procedure may be greatly extended by allowing more than one of the terms $c_{i}-a_{i}$ to be non-zero. We select functions $Y_{1}$ and $y_{0}$ containing one or more parameters so that they have the same functional form but differ in the value of their parameters. Using the recurrence formulas satisfied by these functions one may obtain recurrence formulas for integrals; the first few integrals may be evaluated in the manner illustrated by Eqs. (31) and (32) and the recurrence relations then give all others. This procedure is developed in [B], in which a number of integrals involving the Legendre functions, the confluent hypergeometric function and the Bessel functions are evaluated.

'See Refs. [7] and [8] at the end of [B].

Note added in proof: Work of a similar nature by G. M. Muller has just appeared in Proc. Amer. Math. Soc., Oct. 1954, p. 716. 